\title{
Vibro-Elastography for Visualization of the Prostate Region: Method Evaluation
}

\author{
Seyedeh Sara Mahdavi ${ }^{1}$, Mehdi Moradi ${ }^{1}, \mathrm{Xu} \mathrm{Wen}^{1}$, William J. Morris ${ }^{2}$, \\ and Septimiu E. Salcudean ${ }^{1}$ \\ ${ }^{1}$ Department of Electrical and Computer Engineering, \\ University of British Columbia, Vancouver, Canada \\ tims@ece.ubc.ca \\ ${ }^{2}$ British Columbia Cancer Agency Vancouver, Canada
}

\begin{abstract}
We show that vibro-elastography, an ultrasound-based method that creates images of tissue viscoelasticity contrast, can be used for visualization and segmentation of the prostate. We use MRI as the gold standard and show that VE images yield more accurate 3D volumes of the prostate gland than conventional B-mode imaging. Furthermore, we propose two novel measures characterizing the strength and continuity of edges in noisy images. These measures, as well as contrast to noise ratio, demonstrate the utility of $\mathrm{VE}$ as a prostate imaging modality. The results of our study show that in addition to mapping the visco-elastic properties of tissue, VE can play a central role in improving the anatomic visualization of the prostate region and become an integral component of interventional procedures such as brachytherapy.
\end{abstract}

\section{Introduction}

Segmentation of the prostate is required in prostate cancer treatment. In low dose rate brachytherapy, permanent radioactive seeds must be accurately placed in the prostate and peri-prostatic tissue. In high dose rate brachytherapy, temporary catheters must be repeatedly and accurately placed for radiation fraction delivery. Thus accurate visualization and segmentation of the prostate is important in treatment planning and delivery, and can reduce the possible treatment side effects such as impotence, rectal bleeding, and urinary incontinence.

Image-based guidance for prostate interventions is an active area of research [12]. Ultrasound (B-mode) is the primary imaging modality used for radiation treatment planning and delivery. While safe, accessible and real-time, ultrasound B-mode imaging does not delineate the prostate reliably [3].

In our prior work, we have introduced ultrasound vibro-elastography (VE) to generate patient-specific viscoelastic models of the prostate region, and have shown, based on phantom images and a few patient images, that the method has promise in delineating the prostate and anatomical details such as the urethra [4]. In this paper, we show the effectiveness of VE-based segmentation quantitatively by analyzing data from a more extensive patient study in novel ways. First, we use volume-based measures to compare the overall shape of the 
gland as seen in VE and B-mode images, with MRI images as the gold standard. We then evaluate the performance of VE-based segmentation by computing the contrast to noise ratio (CNR) of the prostate relative to the background in VE and standard B-mode images, and show that VE is vastly superior. Finally, because CNR measures are not appropriate for relatively uniform images that may present strong edges, we propose a third measure based on the strength of the prostate edges. The conventional methods for characterizing edge strength in images include using the maximum of a gradient-based edge detector [5] and measuring the changes in the distribution of image features such as brightness and texture on the two sides of the edge. These approaches have met with little success in the case of ultrasound images due to speckle and image artifacts. Therefore, we propose a new correlation-based index of edge continuity and a model-based statistical approach that relates the edge strength with stationarity of the edge intensity profile.

\section{Vibro-Elastography}

A brachytherapy stepper (EXII, CIVCO Medical Solutions) was modified to enable the acquisition of $3 \mathrm{D}$ vibro-elastography (VE) images during conventional prostate brachytherapy. A shaker was mounted on the transducer cradle in order to vibrate the transducer radially. The cradle rotation was motorized, and a control system and interface were developed to enable the application of compression waves $(0-10 \mathrm{~Hz}$ frequency, $0-3 \mathrm{~mm}$ amplitude) to the rectal wall with probe rotation from -45 to 50 degrees from the sagittal plane. Synchronized with the probe motion, ultrasound B-mode and high-frequency RF data images were acquired from a Sonix RP machine with the sagittal array of a dual plane linear/microconvex broadband $5-9 \mathrm{MHz}$ endorectal transducer (Ultrasonix Medical Corp.). The RF data, collected at the approximate rate of $40 \mathrm{fps}$, was processed to compute the tissue motion resulting from the applied compression. A measure of strain energy was computed in the frequency domain to show tissue stiffness contrast [6]. Our studies involving 14 patients show that the average normalized correlation ( $\mathrm{NC}$ ) of tissue displacement estimation in the vibro-elastography approach is around 0.95 and the average coherence function is over 0.8. This is a clear sign of the reliability of the estimation.

Since elastography measures the mechanical properties of tissue, it can be used for biopsy guidance [7] and to create tissue elasticity models used for needle insertion planning. However, the goal of this paper is to evaluate the VE performance in prostate visualization and segmentation.

\section{Data Acquisition}

The images analyzed in this paper were collected from patients going through the standard prostate brachytherapy procedure at British Columbia Cancer Agency in Vancouver, with additional MR and VE image acquisition. After obtaining informed consent, MR images (slice spacing $4 \mathrm{~mm}$, pixel size $0.27 \times 0.27 \mathrm{~mm}$ ) 

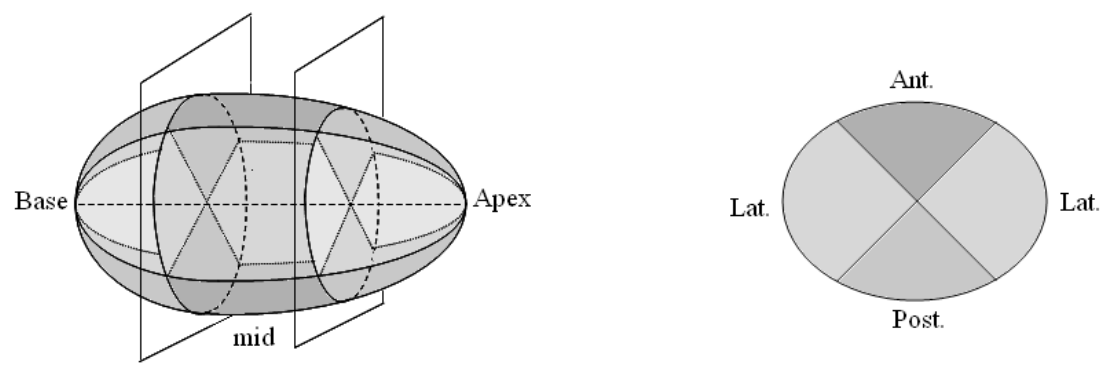

Fig. 1. Transverse planes divide the prostate into base, mid and apex regions. Within each transverse view the prostate is divided to anterior, posterior and lateral sectors.

were collected at the UBC Hospital with a Philips Achieva 3.0 Tesla MRI scanner 1-2 weeks prior to the brachytherapy intervention. A pelvic coil was used for patient comfort and to minimize the deformation of the gland. Transverse Bmode images (slice spacing $5 \mathrm{~mm}$, pixel size $0.156 \times 0.156 \mathrm{~mm}$ ) were obtained as part of the standard treatment planning using an ultrasound machine (B\&K Pro-Focus System B-Series machine with the MFI Biplane Transducer). Intraoperatively, 3D VE images showing the tissue stiffness contrast were acquired at the beginning of the brachytherapy intervention. The sagittal VE images were converted to transverse views via interpolation. For each patient, this process results in 128 transverse images (slice spacing $0.43 \mathrm{~mm}$, pixel size $0.5 \times 0.5 \mathrm{~mm}$ ). So far data from seven patients has been acquired and included in the reported results. MRI images are available from five patients.

\section{Evaluation Methods}

$3 \mathrm{D}$ reconstructions have been generated through delineating the prostate in Bmode, VE and MRI transverse images by an expert. Two types of measures have been used for the evaluation of VE images; volume-based measures and 2D edge/contrast measures. Volume-based measures include "volume difference" and "volume error." 2D edge/contrast measures are the contrast-to-noise ratio, edge strength and edge continuity. The following subsections describe the details of each method. To best represent the critical regions surrounding the prostate, edge evaluation was carried out on the nine distinct regions of the prostate depicted in Figure 1. Division of the prostate in the transverse plane produces three anterior, posterior and lateral sectors and axial division results in the apex, base and mid sectors.

\subsection{Comparison of the Volumes}

Since boundaries of the prostate are more visible in MRI, this modality has been selected as the gold standard. The volume of the prostate gland is an important parameter used in planning the dose distribution in prostate brachytherapy. Volume difference measures the difference between this volume computed from the 
B-mode and VE imaging with that from the gold standard (MRI). Volume error, on the other hand, measures the volume between two registered reconstructed surfaces from VE/B-mode and MRI. Volume error determines how well the extracted "3D shape" from VE/B-mode matches with that of the gold standard.

The VE and B-mode shapes were registered to the MRI by first matching the centers of the volumes and applying a rotation to align the two superiorinferior axes. Then, the point-based Iterative Closest Point (ICP) method [8] was applied to the two surfaces to fine-tune the registration. Due to the clarity of the boundaries in the mid portion of the gland in all three modalities, this region has been selected for volume error calculation. A comparison between the volume-difference of the total gland and volume-error of the mid portion can provide additional information about the base and apex regions.

\subsection{Evaluation of the Contrast - the CNR}

The higher the contrast-to-noise ratio of an image object with respect to the image background, the more distinguishable the object is. To compare the contrast of B-mode and VE images, the CNR was calculated using [9]:

$$
C N R=\frac{2\left(m_{t}-m_{b}\right)^{2}}{\sigma_{t}^{2}+\sigma_{b}^{2}}
$$

in which $m$ and $\sigma^{2}$ are the mean and variance of the target, $t$, and background, $b$, pixel intensities in a region of interest (ROI). The target and background ROI's are selected as regions with the best visible contrast, the target being an area inside the prostate close to the boundary and the background being an area outside the prostate close to the target ROI. Histogram stretching was performed on B-mode and VE images to ensure that the range of intensities in both modalities match.

Because an object may have a low CNR but still be visually distinguishable due to a strong edge, characterization of edges is also required in order to compare the segmentation of the prostate gland in B-mode and VE images.

\subsection{Edge Continuity - A Correlation-Based Measure}

An important characteristic of a good edge is "continuity". We propose a new measure based on the correlation of neighboring edge profiles.

In transversal images, we extended radii in polar coordinates from the center of the prostate. The intersections $r_{\theta_{i}}, i=1, \ldots, N_{\theta}$ of these radii with the boundary of the prostate were manually identified for each $\theta_{i}$, and a radial edge intensity profile function $I_{\theta_{i}}(r)$ was computed for a window $r \in\left[r_{\theta}-\Delta, r_{\theta}+\Delta\right]$. The normalized cross-correlations $R_{\theta_{i} \theta_{i} \pm \delta \theta}(r)$ of $I_{\theta_{i}}(r)$ and $I_{\theta_{i} \pm \delta \theta}(r)$ and the average $c\left(\theta_{i}\right)(r)=\frac{1}{2}\left[R_{\theta_{i} \theta_{i}+\delta \theta}+R_{\theta_{i} \theta_{i}-\delta \theta}\right]$ were computed from these edge intensity profiles. The parameters used in implementation were $\Delta=0.3 \mathrm{~cm}, N_{\theta}=12$ and $\delta \theta=2^{\circ}$. For a strong edge at $\theta_{i}$, the function $c\left(\theta_{i}\right)(r)$ should have a shape similar to a Gaussian distribution with a large peak and small standard deviation indicating high similarity of the two profiles. Thus, we let $P\left(\theta_{i}\right)$ and $\sigma\left(\theta_{i}\right)$ be 

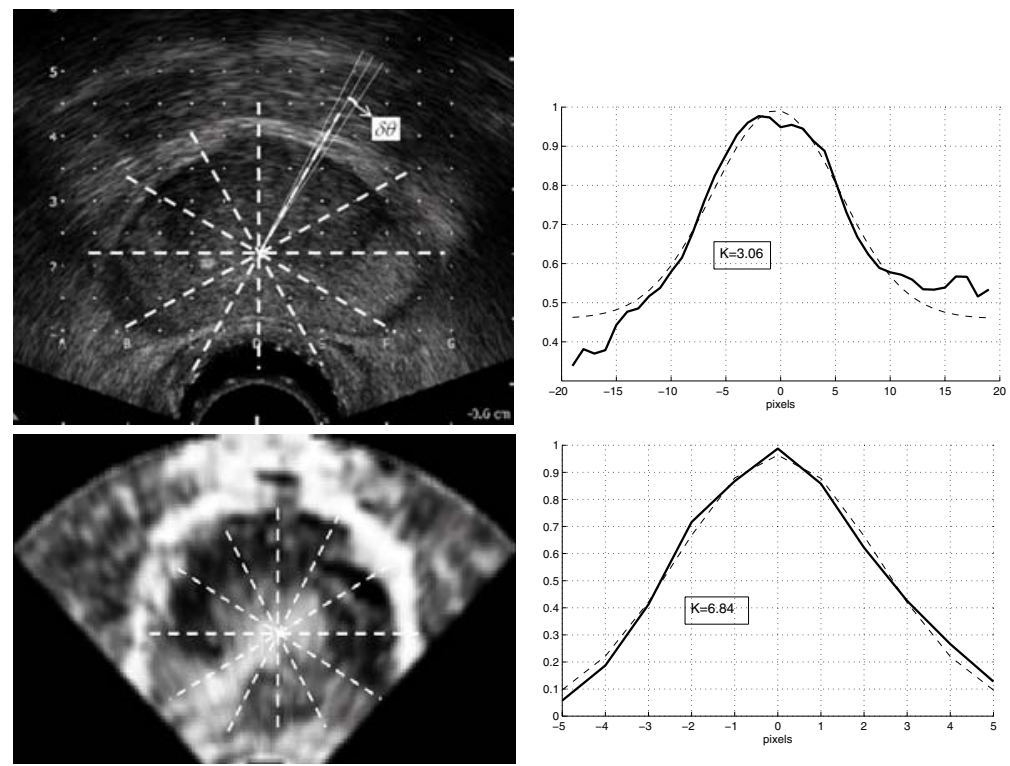

Fig. 2. B-mode (top row) and VE (bottom row) images along with the cross-correlation curve for the specified edge

the peak and standard deviation of a Gaussian function fitted to $c\left(\theta_{i}\right)$ and we define a measure of edge profile consistency, $K\left(\theta_{i}\right)$, as:

$$
K\left(\theta_{i}\right)=\frac{P^{2}\left(\theta_{i}\right)}{\sigma\left(\theta_{i}\right)} .
$$

The average over the dataset of $K$ value was computed for each of the nine regions defined in Figure 1, Figure 2 shows $c\left(\theta_{i}\right)(r)$, the fitted Gaussian function, and the calculated $K$ for a strong edge in both B-mode and VE images.

\subsection{Edge Strength - A Model-Based Statistical Measure}

Apart from being continuous, a good edge should also exhibit high contrast normal to it. Gradient-based edge detectors do not work well in B-mode images as they are plagued by local minima. Therefore, we propose a new approach that models the difference of the radial edge intensity profile as an autoregressive process. The edge strength is characterized based on the degree of stationarity of this process.

In each image, each edge profile $I\left(\theta_{k}\right)(r)$ was considered as a time series $I(i):=I\left(\theta_{k}\right)(\delta r i)$, where the discretized radius distance $i$ replaces the usual time index. For the edge profile $I$, the first order difference is calculated as: $D_{I}(i)=I(i)-I(i-1)$. We model $D_{I}$ as a first order autoregressive $(\operatorname{AR}(1))$ processes of the form:

$$
D_{I}(i)=\phi D_{I}(i-1)+e(i)
$$



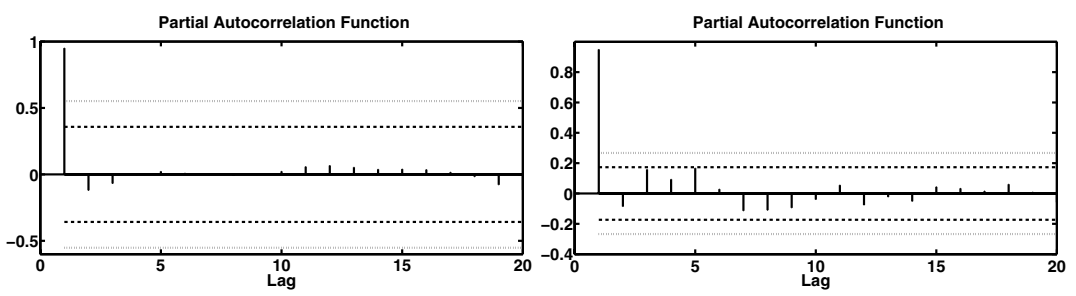

Fig. 3. Typical PACF of the differenced intensity profiles from VE (left) and B-mode (right). The horizontal lines mark the $5 \%$ and $10 \%$ significance levels.

where $e(i)$ is white noise and $\phi$ is the model parameter, estimated based on Yule-Walker equations [10]. The length of the edge profiles is $2 \mathrm{~cm}$. In order to show that an $\mathrm{AR}(1)$ model is sufficient for modeling $D_{I}$, we compute the partial autocorrelation function $(\mathrm{PACF})$ of $D_{I}$. The PACF of an $\mathrm{AR}(1)$ process only has significant values at $\mathrm{lag}=1$. In $78 \%$ of the edge profiles extracted from both B-mode and VE images, at the significance level of 0.05, the PACF function only has significant values at lag=1. Figure 3 shows typical PACF functions for $D_{I}$ extracted from B-mode and VE images.

If a strong edge exists, $D_{I}$ is expected to have a strong peak at the edge. This "trend" of existence of a strong peak means that $D_{I}$ is non-stationary (a signal with a trend can not be stationary, since its statistical moments depend on time, or in our model, on distance). On the other hand, if the radial edge profile does not pass through a strong edge, its derivative is more likely to be trend-free. For an AR(1) model, the condition for stationarity is $|\phi|<1$. Therefore, in our model, larger $|\phi|$ values indicate strong edges. We also performed the Augmented Dickey Fuller (ADF) test [11] to statistically evaluate the edge profiles for their stationarity. The ADF test examines the null hypothesis of non-stationarity against the hypothesis of stationarity.

\section{Results}

Volume measures. Table 1 shows the percentage of volume error (VE/MRI vol. error $\%=100 \times($ non-overlapping vol. from $V E$ and $\left.M R I) /\left(V_{M R I}+V_{V E}\right)\right)$ and volume difference (VE/MRI vol. difference $\left.\%=100 \times\left(V_{V E}-V_{M R I}\right) / V_{M R I}\right)$ between 3D reconstructed prostate shapes from B-mode/MRI and VE/MRI of five patients. The average volume error over the five patients, which is calculated for the mid-region, is similar in both cases $(5.1 \%$ vs. $5.2 \%)$. However, the percentage of volume difference between B-mode and MRI, calculated on the whole volume, is higher than that between VE and MRI ( $5 \%$ vs. $9.6 \%$ ). Since the volume error in the mid sections are fairly similar, the volume differences over the entire gland should be mainly originating from the base and apex.

Contrast evaluation. The CNR of VE and B-mode images, averaged over the three sections of the prostate, is shown in Table 2. In all three regions the CNR of $\mathrm{VE}$ is clearly higher than that of B-mode. 
Table 1. Percentage of vol. error and vol. difference for VE/B-mode and MRI

\begin{tabular}{c||cccccc}
\hline$\%$ & P1 & P2 & P3 & P4 & P5 \\
\hline VE/MRI volume error & 4.0 & 3.0 & 7.9 & 5.6 & 4.4 \\
B-mode/MRI volume error & 6.8 & 2.7 & 5.8 & 4.7 & 6 \\
\hline VE/MRI volume difference & 1.8 & -5.1 & -2.7 & 8.9 & -6.6 \\
B-mode/MRI volume difference & 6.0 & 9.7 & 9.7 & 14.6 & 8.1 \\
\hline
\end{tabular}

Table 2. CNR comparison of VE and B-mode images (data from seven patients)

\begin{tabular}{c||c|c|c}
\hline & Base & Mid & Apex \\
\hline CNR VE & $21.2 \pm 10.2$ & $24.5 \pm 11.6$ & $25.4 \pm 15.2$ \\
CNR B-mode & $4.8 \pm 1.9$ & $1.3 \pm 0.6$ & $1.8 \pm 1.2$ \\
\hline
\end{tabular}

Table 3. Edge continuity measure for nine sectors of the prostate (seven patients)

\begin{tabular}{l||c|c|c||c|c|c}
\hline & Base & Mid & Apex & Base & Mid & Apex \\
\hline Ant. & $1.43 \pm 1.56$ & $1.29 \pm 1.1$ & $0.67 \pm 0.79$ Ant. & $0.78 \pm 0.64$ & $1.03 \pm 0.82$ & $0.78 \pm 0.54$ \\
Lat. & $0.86 \pm 0.88$ & $1.11 \pm 0.94$ & $0.48 \pm 0.57$ Lat. & $0.69 \pm 0.58$ & $0.94 \pm 0.71$ & $0.75 \pm 0.65$ \\
Post & $0.85 \pm 0.73$ & $0.87 \pm 0.81$ & $0.38 \pm 0.54$ Post & $0.34 \pm 0.38$ & $0.33 \pm 0.41$ & $0.52 \pm 0.44$ \\
\hline
\end{tabular}

(a) B-mode

(b) $\mathrm{VE}$
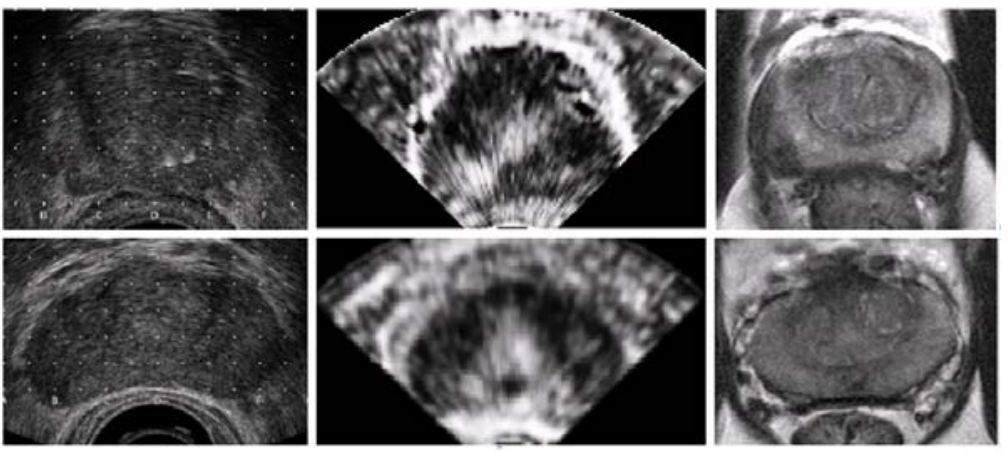

Fig. 4. B-mode, VE and MRI sample images from two patients

Edge continuity: Tables $[3$ and $[3$ b show the mean and standard deviation of the edge continuity measure, $K$, for nine sectors of the prostate. The B-mode and VE images of seven patients have been used. The overall mean value of $K$ for VE images is larger than that of B-mode in the apex of the gland. However, $K$ values for $\mathrm{VE}$ images degrade in the mid and base sections.

Edge strength based on the AR model and ADF test. The absolute value of $\operatorname{AR}(1)$ coefficient $|\phi|$ for the difference edge profiles $\left(D_{I}\right)$ was significantly larger in VE images than in B-mode images in all nine areas $(|\phi|=0.59 \pm 0.19$ for VE images and $|\phi|=0.19 \pm 0.11$ for B-mode images). The ADF test shows 
that in VE images, the stationarity hypothesis is rejected $(p<0.1)$ for $94 \%$ of the edge profiles. Whereas, in B-mode images, the stationarity hypothesis is rejected only in $23 \%$ of the profiles. In other words, more than $77 \%$ of the Bmode edge profiles are stationary, suggesting that there is no strong trend in the profile which goes against having a strong edge.

\section{Discussion and Conclusions}

In this paper, vibro-elastography (VE) is evaluated as an imaging modality to visualize and segment prostate. A visual comparison of VE and B-mode in more than 20 patients scanned so far clearly shows that VE imaging is a promising modality for prostate interventions (Figure 4). Quantitative results presented in this paper also confirm this observation. We showed that VE is successful in extracting the 3D shape of the prostate specially in the base and apex region, with MRI as the gold standard. The regional CNR of VE images is significantly higher than that of B-mode. In order to compare the edge qualities in B-mode and VE images, novel measures have been proposed that show stronger edges in VE images, characterized by non-stationarity of the edge profiles in VE modality. Our proposed measure of edge continuity indicates more consistent edges, compared to B-mode, in VE images of the apex region. In other areas, specially in the posterior region near the transducer, VE images deteriorate. Potential causes to this problem are transducer slip, which deteriorates the image close to the probe, and the use of only axial strain estimation [6] in determining prostate elasticity. This can generate known "softening" artifacts. Understanding this shortcoming and the visualization of other anatomical details, e.g. urethra and the cavernosal nerve plexi, are the subject of future research. Also, as a future goal, we plan to carry out a more thorough investigation of the proposed measures of edge strength and continuity on simulated ultrasound data.

Acknowledgments. We would like to thank Dr. T. Pickles, Dr. M. McKenzie, the staff at the BC Cancer Agency, Mr. O. Goksel and Mr. R. Zahiri Azar. Financial support from NIH grant R21 CA120232-01 is gratefully acknowledged.

\section{References}

1. Jain, A., Deguet, A., Iordachita, I., Chintalapani, G., Blevins, J., Le, Y., Armour, E., Burdette, C., Song, D., Fichtinger, G.: Intra-operative 3D guidance in prostate brachytherapy using a non-isocentric c-arm. Med. Image Comput. Comput. Assist Interv. Int. Conf. Med. Image Comput. Comput. Assist. Interv. 10(Pt 2), 9-17 (2007)

2. Wei, Z., Ding, M., Downey, D., Fenster, A.: 3D TRUS guided robot assisted prostate brachytherapy. Med. Image Comput. Comput Assist. Interv. Int. Conf. Med. Image Comput. Comput. Assist. Interv. 8(Pt 2), 17-24 (2005)

3. Smith, S., Wallner, K., Merrick, G., Butler, W., Sutlief, S., Grimm, P.: Interpretation of pre- versus postimplant TRUS images. Med. Phys. 30(5), 920-924 (2003) 
4. Salcudean, S.E., French, D., Bachmann, S., Zahiri-Azar, R., Wen, X., Morris, W.J.: Viscoelasticity modeling of the prostate region using vibro-elastography. Med. Image Comput. Comput. Assist. Interv. Int. Conf. Med. Image Comput. Comput. Assist. Interv. 9(Pt 1), 389-396 (2006)

5. Canny, J.: A computational approach to edge detection. IEEE Transactions on Pattern Analysis and Machine Intelligence 8(6), 679-698 (1986)

6. Zahiri-Azar, R., Salcudean, S.E.: Motion estimation in ultrasound images using time domain cross correlation with prior estimates. IEEE Trans. Biomed. Eng. 53(10), 1990-2000 (2006)

7. Pesavento, A., Lorenz, A.: Real time strain imaging and in-vivo applications in prostate cancer. In: Proc. IEEE Ultrasonics Symposium, vol. 2, pp. 1647-1652 (2001)

8. Besl, P.J., McKay, H.D.: A method for registration of 3D shapes. IEEE Trans. Pattern Analysis and Machine Intelligence 14(2), 239-256 (1992)

9. Bilgen, M., Insana, M.F.: Predicting target detectability on acoustic elastography. In: IEEE Ultrasonics Symposium, pp. 1427-1430 (1997)

10. Shumway, R.H., Stoffer, D.S.: Time Series Analysis and Its Applications: With R Examples. Springer Texts in Statistics. Springer, Heidelberg (2006)

11. Dickey, D.A., Fuller, W.A.: Distribution of the estimators for autoregressive time series with a unit root. Journal of the American Statistical Association 74(366), 427-431 (1979) 